\title{
Soccer Post-Match Recovery - a Review of the Evidence on Static Stretching
}

\author{
Dr. Ryan Sciacchitano, ND
}

Canadian College of Naturopathic Medicine

\begin{abstract}
Soccer is the most played sport worldwide, with over 265 million participants ${ }^{1}$. It is an incredibly demanding sport, with many different technical skills and physical loads placed on the body. This makes post-match recovery strategies amongst high level soccer players of great importance. The aim of this review is to summarize the existing literature on stretching for post-match recovery, examining its relation to injury prevention, Delayed Onset Muscle Soreness (DOMS), and performance. Scientific evidence of the highest quality and relevance was extracted and reviewed. Despite its common practice, evidence does not support static stretching as a modality to improve recovery post-match amongst soccer players. Larger trials with important outcome measures are needed to determine if a post-match stretching regimen to facilitate recovery exists.
\end{abstract}

Keywords: Static; Stretching; Post-Match; Soccer; Recovery; DOMS; Injury; Performance

\section{Introduction}

Soccer is the most played sport worldwide, with over 265 million participants. ${ }^{1}$ It is an incredibly demanding sport, with many different technical skills and physical loads placed on the body leading to the potential for injury. Across many different regions for various reasons, one of which being climate, game schedules become condensed which can further predispose athletes to injury. When less than 96 hours separates 2 matches, the risk of injury is widely believed to increase, ${ }^{2,3}$ with an estimated 6 -fold increase when 2 games are played in the same week. ${ }^{4}$ When 3 games are played in a week, many detrimental concerns have been observed, including reduced sprint times, increased muscle soreness, impaired knee range of motion, increased muscle damage, increased oxidative stress, and elevated inflammatory markers. ${ }^{5}$ Thus, many strategies are being implemented to help mitigate these effects, one of which being stretching. The aim of this review is to summarize the existing literature on static stretching for post-match recovery and provide practical evidence-based recommendations that can be implemented by coaches and trainers to help facilitate athlete recovery.

\section{Materials and Methods}

PubMed was utilized as a tool to research existing published data in relation to stretching for postmatch recovery amongst soccer players. The study keywords were used as MeSH terms with various filters utilized in combination to extract evidence of the highest relevance and quality. Searches were conducted specifically amongst human populations, with preference given to research considered to be of a higher level of evidence. These include Meta-analyses, Systematic Reviews, and Randomized Controlled Trials.

\section{Results}




\section{Static stretching background}

Static stretching is theorized to increase range of motion through maintaining a passive lengthened position for an extended period of time. ${ }^{6}$ While other forms of stretching exist (i.e. Dynamic Stretching, Proprioceptive Neuromuscular Facilitation or PNF, etc.), this review will focus exclusively on static stretching as that is what is often implemented post-match amongst soccer players. A review of 32 practitioners implementing recovery strategies amongst French professional soccer teams discovered stretching was utilized by $50 \%$ of them. ${ }^{7}$ This split elucidates the controversy that currently exists within the soccer community on stretching and its benefits on recovery. Many variables can be individually debated, including which stretches are best, the length of time a stretch should be held, number of times it should be done, intensity of the stretch, etc. ${ }^{8,9}$ This inherently makes stretching a difficult topic to study, reflected by a dearth of quality published literature on the topic. Nevertheless, a summary is compiled below in regards to stretching and post-match recovery. Specifically, the effect of stretching on injury prevention, Delayed Onset Muscle Soreness (DOMS), and performance are reviewed.

\section{Injury prevention}

Static stretching regimens have been shown to improve musculotendinous stiffness ${ }^{10,11}$ as well as decrease injury rates amongst football players. ${ }^{9,12,13,14} \mathrm{~A}$ high intensity sport such as soccer requires a sufficiently compliant muscle-tendon unit to absorb and release a significant amount of energy, which routine static stretching is thought to improve. ${ }^{15}$ This has led to the theory that implementing stretches post-match, when the muscles are thoroughly warmed-up and malleable, will help with injury prevention in subsequent matches. To the contrary, the theory that incurring more muscle damage through stretching post-match also exists, in that lengthening a muscle that has just undergone significant damage is counterproductive. Examining existing literature, a specific post-match protocol has not been identified. In fact, multiple systematic reviews demonstrated no benefit on injury risk. ${ }^{16,17} \mathrm{~A}$ Cochrane review concluded that there is not enough evidence to either recommend or discontinue static stretching post-exercise for the purposes of reducing injury rates. ${ }^{18}$

\section{Delayed Onset Muscle Soreness}

DOMS is a phenomenon that can occur at varying intensities after vigorous exercise, unaccustomed exercise, or eccentric training where the muscle is under tension while lengthening. ${ }^{19}$ It is of particular concern for soccer players as it can negatively affect subsequent performances through decreasing range of motion at major joints, as well as reducing shock attenuation and peak torque. ${ }^{20}$ Further, unaccustomed stresses can be placed on muscles and tendons while suffering from DOMS, leading to an increased risk of injury. ${ }^{20} \mathrm{~A}$ Cochrane review summarizing numerous Randomized Controlled Trials demonstrated stretching after exercise did not produce clinically relevant outcomes in DOMS amongst healthy adults. ${ }^{18}$ Of the studies mentioned, only one was specifically related to soccer. ${ }^{21}$ That study was excluded from the Cochrane review because it was not clearly randomised, and it combined stretching with jogging and a "shake-down". A further search of the literature identified no studies related to postmatch stretching reducing DOMS amongst soccer players.

\section{Performance}

Optimizing ranges of motion, usually related to those at the hips, knees, and ankles, can improve various movements required to perform at a high level during soccer. ${ }^{22,23}$ Attaining and regaining those ranges 
of motion post-match are considered important factors for subsequent match performance. The most soccer-relevant study to date compared an active recovery protocol (12 minutes submaximal running, 8 minutes static stretching) to a passive recovery protocol (20 minutes sitting) amongst 31 professional soccer players post-training. ${ }^{24}$ During a subsequent training session (24 hours later) there were no significant differences observed in the Rate of Perceived Exertion (RPE), heart rate, lower limb flexibility, $20 \mathrm{~m}$ sprint, and the Balsom agility test. However, the counter-movement jump did show statistically significant improvement in the active recovery group. ${ }^{24}$ While positive, this benefit could be explained more-so by the submaximal running portion of the recovery protocol as opposed to the static stretching portion. Other studies have supported other methods of recovery, including submaximal cardiovascular work, over static stretching in benefitting subsequent soccer performances. ${ }^{25,26,27}$

\section{Discussion}

Despite its common practice, existing literature does not support the recommendation for stretching as part of the soccer post-match routine. Contributing to the lack of evidence are the numerous variables to control for while researching stretching, including intensity and position. ${ }^{8,9}$ It has yet to be determined if an optimum level of post-match static stretching to prevent injury, reduce DOMS, and improve performance exists amongst soccer players. If it does exist, larger trials with important outcome measures are needed to determine a regimen of how to best attain the benefits. For the time being, high level teams should focus their recovery on more evidence based approaches, including nutritional and hydration strategies, optimizing sleep, and cold water immersion. ${ }^{7}$

Funding: This research received no external funding.

Conflicts of Interest: The author declares no conflict of interest 


\section{References}

1. Kunz, M. 265 million playing football. FIFA Magazine. 2007. Retrieved from: https://www.fifa.com/mm/document/fifafacts/bcoffsurv/emaga_9384_10704.pdf.

2. Lago-Peñas C, Rey E, Lago-Ballestexros J, Casáis L, Domínguez E. The influence of a congested calendar on physical performance in elite soccer. J Strength Cond Res. 2011; 25(8):2111-7. DOI:

10.1519/JSC.0b013e3181eccdd2.

3. Carling C, Le Gall F, Dupont G. Are physical performance and injury risk in a professional soccer team in match-play affected over a prolonged period of fixture congestion? Int J Sports Med. 2012; 33(01):3642. DOI: 10.1055/s-0031-1283190.

4. Dupont G, Nedelec M, McCall A, McCormack D, et al. Effect of 2 soccer matches in a week on physical performance and injury rate. Am J Sports Med. 2010; 38(9):1752-8. DOI: 10.1177/0363546510361236.

5. Mohr M, Draganidis D, Chatzinikolaou A, Barbero-Álvarez JC, et al. Muscle damage, inflammatory, immune and performance responses to three football games in 1 week in competitive male players. Eur J Appl Physiol. 2016; 116(1):179-93. DOI: 10.1007/s00421-015-3245-2.

6. Lewis J. A systematic literature review of the relationship between stretching and athletic injury prevention. Orthop Nurs. 2014; 33(6):312-20. DOI: 10.1097/NOR.0000000000000097.

7. Nédélec M1, McCall A, Carling C, Legall F, et al. Recovery in soccer: part ii-recovery strategies. Sports Med. 2013; 43(1):9-22. DOI: 10.1007/s40279-012-0002-0.

8. Dadebo B, White J, George KP. A survey of flexibility training protocols and hamstring strains in professional football clubs in England. Br J Sports Med. 2004; 38(4):388-94.

9. Apostolopoulos N, Metsios GS, Flouris AD, Koutedakis Y, et al. The relevance of stretch intensity and position-a systematic review. Front Psychol. 2015; 18(6):1128. DOI: 10.3389/fpsyg.2015.01128.

10. Bandy WD, Irion JM, Briggler M. The effect of time and frequency of static stretching on flexibility of the hamstring muscles. Phys Ther. 1997;7 7(10):1090-6.

11. Kay AD, Blazevich AJ. Reductions in active plantarflexor moment are significantly correlated with static stretch duration. Eur J Sport Sci. 2008;8(1):41-6. DOI: 10.1152/japplphysiol.91476.2008.

12. Witvrouw E, Danneels L, Asselman P, et al. Muscle flexibility as a risk factor for developing muscle injuries in male professional soccer players: a prospective study. Am J Sports Med. 2003;31(1):41-6. 
13. McHugh MP, Cosgrave $\mathrm{CH}$. To stretch or not to stretch: the role of stretching in injury prevention and performance. Scand J Med Sci Sports. 2010; 20(2):169-81. DOI: 10.1111/j.1600-0838.2009.01058.x.

14. Bradley PS, Portas MD. The relationship between preseason range of motion and muscle strain injury in elite soccer players. J Strength Cond Res. 2007; 21(4):1155-9.

15. Witvrouw E, Mahieu N, Danneels L, McNair P. Stretching and injury prevention: an obscure relationship. Sports Med. 2004; 34(7):443-9.

16. Hart, L. Effect of stretching on sport injury risk: a review. Clin J Sport Med. 2005 Mar; 15(2):113.

17. Torres R, Ribeiro F, Alberto Duarte J, Cabri JM. Evidence of the physiotherapeutic interventions used currently after exercise-induced muscle damage: systematic review and meta-analysis. Phys Ther Sport. 2012; 13(2):101-14. DOI: 10.1016/j.ptsp.2011.07.005.

18. Herbert RD, de Noronha M, Kamper SJ. Stretching to prevent or reduce muscle soreness after exercise. Cochrane Database Syst Rev. 2011; 6(7):CD004577. DOI: 10.1002/14651858.CD004577.pub3.

19. Ranchordas MK1, Rogerson D, Soltani H, Costello JT. Antioxidants for preventing and reducing muscle soreness after exercise. Cochrane Database Syst Rev. 2017; 12:CD009789. DOI:

10.1002/14651858.CD009789.pub2.

20. Cheung K1, Hume P, Maxwell L. Delayed onset muscle soreness: treatment strategies and performance factors. Sports Med. 2003; 33(2):145-64.

21. Reilly T, Rigby M. Effect on active warm-down following competitive soccer. Science and Football IV. 2002; 226-229.

22. Akbulut T, Agopyan A. Effects of an Eight-Week Proprioceptive Neuromuscular Facilitation Stretching Program on Kicking Speed and Range of Motion in Young Male Soccer Players. J Strength Cond Res. 2015; 29(12):3412-23. DOI: 10.1519/JSC.0000000000001015.

23. Amiri-Khorasani M, Abu Osman NA, Yusof A. Acute effect of static and dynamic stretching on hip dynamic range of motion during instep kicking in professional soccer players. J Strength Cond Res. 2011; 25(6):1647-52. DOI: 10.1519/JSC.0b013e3181db9f41.

24. Ezequiel Rey, Carlos Lago-Peñas, Luis Casáis, and Joaquín Lago-Ballesteros. The Effect of Immediate Post-Training Active and Passive Recovery Interventions on Anaerobic Performance and Lower Limb Flexibility in Professional Soccer Players. J Hum Kinet. 2012; 31:121-129. DOI: 10.2478/v10078-0120013-9.

25. Kinugasa T, Kilding AE. A comparison of post-match recovery strategies in youth soccer players. $J$ Strength Cond Res. 2009; 23(5):1402-7. DOI: 10.1519/JSC.0b013e3181a0226a.

26. Tessitore A, Meeusen R, Cortis C, Capranica L . Effects of different recovery interventions on anaerobic performances following preseason soccer training. J Strength Cond Res. 2007; 21(3):745-50. 
27. Andersson H, Raastad T, Nilsson J, Paulsen G, et al. Neuromuscular fatigue and recovery in elite female soccer: effects of active recovery. Med Sci Sports Exerc. 2008; 40(2):372-80. DOI:

10.1249/mss.0b013e31815b8497. 